\title{
Strategies for estimating human exposure to mycotoxins via food
}

\author{
M. de Nijs ${ }^{1 *}$, M.J.B. Mengelers ${ }^{2}$, P.E. Boon ${ }^{2}$, E. Heyndrickx ${ }^{3}$, L.A.P. Hoogenboom ${ }^{1}$, P. Lopez ${ }^{1}$ and H.G.J. Mol $^{1}$ \\ ${ }^{1}$ RIKILT, Wageningen UR, P.O. Box 230, 6700 AB Wageningen, the Netherlands; ${ }^{2}$ National Institute for Public Health \\ and the Environment (RIVM), P.O. Box 1, 3729 BA Bilthoven, the Netherlands; ${ }^{3}$ Laboratory of Food Analysis, Faculty of \\ Pharmaceutical Sciences, Ghent University, Ottergemsesteenweg 460, 9000 Ghent, Belgium; monique.denijs@wur.nl
}

Received: 2 February 2016 / Accepted: 24 July 2016

(c) 2016 Wageningen Academic Publishers

\section{OPEN ACCESS @(1)(-) REVIEW ARTICLE}

\begin{abstract}
In this review, five strategies to estimate mycotoxin exposure of a (sub-)population via food, including data collection, are discussed with the aim to identify the added values and limitations of each strategy for risk assessment of these chemicals. The well-established point estimate, observed individual mean, probabilistic and duplicate diet strategies are addressed, as well as the emerging human biomonitoring strategy. All five exposure assessment strategies allow the estimation of chronic (long-term) exposure to mycotoxins, and, with the exception of the observed individual mean strategy, also acute (short-term) exposure. Methods for data collection, i.e. food consumption surveys, food monitoring studies and total diet studies are discussed. In food monitoring studies, the driving force is often enforcement of legal limits, and, consequently, data are often generated with relatively high limits of quantification and targeted at products suspected to contain mycotoxin levels above these legal limits. Total diet studies provide a solid base for chronic exposure assessments since they provide mycotoxin levels in food based on well-defined samples and including the effect of food preparation. Duplicate diet studies and human biomonitoring studies reveal the actual exposure but often involve a restricted group of human volunteers and a limited time period. Human biomonitoring studies may also include exposure to mycotoxins from other sources than food, and exposure to modified mycotoxins that may not be detected with current analytical methods. Low limits of quantification are required for analytical methods applied for data collection to avoid large uncertainties in the exposure due to high numbers of left censored data, i.e. with levels below the limit of quantification.
\end{abstract}

Keywords: exposure assessment, food consumption, mycotoxins, human biomonitoring, total diet study, duplicate diet study, food monitoring

\section{Introduction}

Food safety authorities aim at protecting the health of consumers by taking measures to avoid exposure of the population to harmful levels of these compounds. The measures should ensure that exposure of the population is below health-based guidance values, such as an acute reference dose or tolerable daily or weekly intake. The measures are preferably based on science-based risk evaluations from competent food safety authorities. Risk assessment studies, composed of hazard identification, characterisation and exposure assessment, are an integral part of these evaluations (Brera et al., 2014; FAO/WHO, 2012; Marin et al., 2013; Van Egmond et al., 2007).
Food safety authorities of EU Member States are obliged to monitor the occurrence of harmful compounds in food by implementing risk-based monitoring programs (EC, 2004). Results from long-term monitoring of the occurrence of undesirable substances in food can also provide input for a trend analysis on levels and exposure in order to evaluate the effectiveness of implemented measures, to discover unknown sources of mycotoxins, and to give insight in changing trends in contamination of crops.

Food can be contaminated with a mixture of mycotoxins, natural toxins produced by fungal species in all parts of the world. Fungal invasion and mycotoxins production can take place both in the field and/or during storage of the crops (Han et al., 2014). The actual contamination 
levels are determined by complex interaction among fungal species, plant cultivars and environmental factors (Battilani et al., 2012; Van der Fels-Klerx, 2014). The occurrence of fungi on crops varies between geographic regions, climatic conditions and pre- and post-harvest management (De Rijk et al., 2015). The types and levels of mycotoxins in food can therefore vary considerably. Industrial processes and household preparation processes, such as cleaning or peeling, may furthermore alter the profile and the levels of mycotoxins in consumed food (Cano-Sancho et al., 2013; Meca et al., 2013; Tittlemier et al., 2014; Vidal et al., 2014).

International trade of food and feed adds variation to the exposure to mycotoxins and introduces extra challenges to assess the exposure (Cressey, 2009; Kendra and Dyer, 2007). Additionally, dietary exposure varies between geographic regions due to variability in the diets consumed and specific individual consumption patterns (Boon et al., 2009; Brera et al., 2014; EFSA, 2014b; FAO/WHO, 2012).
The present paper will describe and discuss five strategies applied in exposure assessments of mycotoxins. First, four data collection studies are described, followed by the description of the use of these data in four well established exposure assessment strategies. Secondly, a new strategy for exposure assessment, human biomonitoring, is described. Benefits and limits of all five exposure assessment strategies are discussed in the final chapter.

\section{Data collection studies for exposure assessment}

Application of the four well-established exposure assessment strategies described in Section 3, for the assessment of the dietary exposure to mycotoxins, require data on food consumption as well as data on levels of mycotoxins in food. Table 1 presents a schematic overview of the food consumption and data collection studies for these wellestablished exposure assessment strategies.

Table 1. Characteristics of: (1) study design; (2) sample number and analysis; (3) ethical issues; (4) costs; and (5) ability to use provided samples for other contaminants.

\begin{tabular}{|c|c|}
\hline Data collection study & Characteristics \\
\hline $\begin{array}{l}\text { Food consumption surveys } \\
\text { (FCSs) }\end{array}$ & $\begin{array}{l}\text { 1. Complex study design. } \\
\text { 2. Large number of subjects required, including subgroups in the population. } \\
\text { 3. Volunteers involved, requiring ethical approval and management of privacy data. } \\
\text { 4. High costs for collection of data and processing of data. } \\
\text { 5. Data can be used for exposure assessment to food chemicals and intake assessment to nutrients, both acute and } \\
\text { chronic (if covering more than one day per subject). }\end{array}$ \\
\hline Food monitoring studies & $\begin{array}{l}\text { 1. Simple study design. } \\
\text { 2. Large number of analytical samples, often from routine monitoring (official control), often analysed with multi- } \\
\text { methods with relatively high limits of quantification or reporting limits; no dilution of samples. } \\
\text { 3. No volunteers involved. } \\
\text { 4. High costs for sample collection, relatively low analysis costs per sample/mycotoxin. Primary aim in general is } \\
\text { compliance checks, data from this can be used for exposure assessment studies without additional costs. } \\
\text { 5. Samples can be used for analysis of other food chemicals and to estimate acute and chronic exposure to these } \\
\text { compounds. }\end{array}$ \\
\hline Total diet studies & $\begin{array}{l}\text { 1. Initial study design complex but can be re-used in follow-up studies. } \\
\text { 2. Samples are pooled per food category, resulting in dilution of higher contaminated samples; sensitive analysis } \\
\text { required to achieve quantifiable results. } \\
\text { 3. No volunteers involved; possibly ethical approval required when investigating diets of ethical groups in the } \\
\text { population. } \\
\text { 4. High costs for the initial design of the study; high costs for sample collection, preparation and analysis. } \\
\text { 5. Samples can be used for other food chemicals but only when the study design is suitable for this topic (so called } \\
\text { total diet-like studies); only allows estimation of chronic exposure to contaminants. }\end{array}$ \\
\hline Duplicate diet studies & $\begin{array}{l}\text { 1. Simple study design. } \\
\text { 2. Limited number of samples because all foods are pooled into one sample and small sample size; sensitive analysis } \\
\text { required to achieve quantifiable results. } \\
\text { 3. A representative group of reliable volunteers must be identified; ethical approval is required and privacy must be } \\
\text { managed; sample collection puts a high burden on the volunteers. } \\
\text { 4. High costs for sample preparation; high costs for studies; high costs for processing of food diaries. } \\
\text { 5. Samples may be used for analysis of chemicals in food; allows estimation of chronic (if covering more than one day } \\
\text { per subject) and acute exposure to contaminants. }\end{array}$ \\
\hline
\end{tabular}




\section{Food consumption surveys}

The amount of a single food ingredient consumed influences the potential dietary exposure of humans to mycotoxins (Brera et al., 2014; Cressey and Reeve, 2013; EFSA, 2014b; O'Mahony and Vilone, 2013). Data on food consumption of a population are collected via questionnaires from volunteers and, ideally, at the individual level, at a high level of detail, and covering at least two days. At a national level, such databases are widely used to assess the dietary exposure to mycotoxins by the population (Boon et al., 2009; Sprong et al., 2016b). At the international level, various databases are available for exposure assessment. The situation in the EU is assessed by the European Food Safety Authority (EFSA), while the Food and Agriculture Organisation (FAO) of the United Nations evaluates the global food situation, including countries with insufficient food supplies (EFSA, 2015a; FAO/WHO, 2015).

In 2011, EFSA introduced the Comprehensive European Food Consumption Database, hereafter called EFSA Comprehensive Database (EFSA, 2011d). The various EU Member States provide(d) EFSA with data from their most recent national dietary surveys, at the level of the individual consumer. In addition, the food classification system 'FoodEx1' was developed by EFSA to codify all foods and beverages present in the database. FoodEx1 is a hierarchical system based on 20 main food categories that are further divided into subgroups up to a maximum of 4 levels. In 2015, the EFSA Comprehensive Database was updated with additional, up-to-date, food consumption data (EFSA, $2015 b)$. These data were coded with an upgraded version of EFSA's food classification and description system, FoodEx2, which enables more precise reporting of consumption patterns. The FoodEx1 database, up to now used by EFSA, includes food consumption data concerning infants, toddlers, children, adolescents, adults, elderly and very elderly from 32 different dietary surveys carried out in 22 different Member States. Summary statistics are calculated and are available at the EFSA website (EFSA, 2015a). These statistics include the total number of individuals per survey and age class and, for all four levels of FoodEx1, per age class and survey, the total number of consumers, and the mean, the median and the standard deviation of the consumption, as well as low and high percentiles. Food consumption statistics are reported both in grams per day and in grams per $\mathrm{kg}$ body weight per day, for both chronic and acute consumption, and for both the total population and consumers only (i.e. those that reported consumption of the specific food product on the one or more days included in the survey). These summary statistics can be used as a quick screening tool to assess chronic and acute exposure to hazardous substances. EFSA uses the detailed underlying consumption data at the individual level to perform more refined exposure assessments, both acute and chronic.
To assess global food intake, the WHO has managed since 1976 a Food Contamination Monitoring and Assessment Programme, within the Global Environment Monitoring System, commonly known as GEMS/Food (WHO, 2015). WHO implements the programme in cooperation with a network of Collaborating Centres and recognised national institutions located all around the world. As part of its dietary exposure assessment mandate, GEMS/Food has developed supra-national model diets that are currently used for estimating dietary intake of various chemicals according to internationally accepted methodologies. Guidance documents have been developed for International Estimated Short-term Intake (IESTI) (FAO/WHO, 2014), International Estimated Daily Intake (IEDI) and a harmonised Total Diet Study approach (see next section) (EFSA/FAO/WHO, 2011b). A monograph on the principles and methods for the risk assessment of chemicals in food has been published (FAO/WHO, 2009).

WHO and FAO have recently developed a new database for Individual Food Consumption Data. This Chronic Individual Food Consumption database - Summary statistics (CIFOCOss), is hosted by WHO and currently contains summary statistics of 37 surveys from 26 countries (only surveys with a survey duration of two days or more) (WHO, 2015). It has been developed to be used by FAO/ WHO scientific committees in particular for dietary exposure assessment purposes. It provides summary statistics at three levels of food categorisation and can therefore only be used for a rough indication of the dietary exposure at national level. CIFOCOss is continuously being expanded with data from additional food consumption surveys (WHO, 2015).

\section{Data collection studies}

Occurrence data on mycotoxins collected for exposure assessment purposes always require low limits of detection (LOQs) to prevent left-censored data or non-detects ('below LOQ'). The assumption that the levels of mycotoxins in the samples with left censored data are as high as the LOQ, the upper bound principle, only allows worst case estimates (EFSA, 2010). This approach suffices if the margin between assumed exposure levels and levels causing the toxic effects is sufficiently large, but this is often not the case for mycotoxins. EFSA identified this problem with the genotoxic carcinogen sterigmatocystin (EFSA, 2013b) for which the upper bound approach led to a low margin of exposure, as a result from the fact that virtually all available data were left censored due to high LOQs. EFSA calculated a required LOQ of $1.5 \mu \mathrm{g} / \mathrm{kg}$ : based on consumption of the relevant food products, the level causing adverse effects in laboratory animals and the minimal required margin of exposure for genotoxic carcinogens. A dedicated survey was subsequently performed at an LOQ of $0.5 \mu \mathrm{g} / \mathrm{kg}$ and sterigmatocystin was detected in $10 \%$ of the 1,259 samples, 
in most cases below $1 \mu \mathrm{g} / \mathrm{kg}$ (Mol et al., 2015). Besides the issue of low LOQs, another issue with certain mycotoxins is the high heterogeneity of the contamination which may result in a high uncertainty of the analysis results (higher than the measurement uncertainty of the analysis as such), but this clearly depends on the type of food product.

\section{Food monitoring study}

Food monitoring studies investigate the prevalence and concentration of contaminants, including mycotoxins, in single food ingredients or foods (Lopez et al., 2016b; Schwartzbord and Brown, 2015; Straumfors et al., 2015). This increases the chance of detecting the mycotoxins under investigation.

Samples in monitoring studies are typically collected randomly at various points in the supply chain, ideally assuring that a food product can be traced to the producer and repeatedly sampled over a longer time period. The aim of sampling within national (long-term) monitoring programs often is to check for compliance or law enforcement in risk-based designed studies (EC, 2004). This allows higher LOQs, often relatively close to the maximum legal limits (Sulyok et al., 2010). This may compromise the use of the data in exposure assessments, since samples taken in targeted studies may reflect higher occurrence and/or levels of mycotoxins than to which the consumer is exposed. The aim of the sampling should be clearly indicated to prevent potential bias. In addition, samples can also be collected in surveys (De Nijs et al., 2013; Lopez et al., 2016b; Sanders et al., 2014), certainly in case of nonregulated mycotoxins. Results of such surveys are frequently published in literature, contrary to data from long-term monitoring programmes (Muller and Korn, 2013).

The relevance of occurrence data from food monitoring studies for exposure assessments is determined by the information accompanying the samples. Classification and description are important to derive exposure estimations for the general population as well as for sensitive groups in the population, such as infants or people with specific diets as in the case of celiac disease (EFSA, 2013c). Knowledge on geographic origin is of interest for trend analysis and for adjustments to risk-based monitoring programs. Sample size, sampling strategy and sample preparation must be documented since these may influence the exposure results (De Rijk et al., 2015; Garcia-Cela et al., 2013; Pichler, 2013; Spanjer et al., 2006).

In addition to confirmatory methods, also relatively simple screening methods like immunoassays may be used in food monitoring studies. Screening methods are by definition unsuitable to identify the target compound but can be used to exclude their presence above a certain cut-off value.
Data obtained with such screening methods require careful evaluation before use in exposure assessments.

Food monitoring studies analyse mycotoxin levels often in raw food ingredients. Occurrence data from these sources require a processing factor when used in exposure assessments, to translate the levels to contamination levels in the ready-to-eat foods. Mycotoxins can be lost after peeling (e.g. vegetables), cleaning and sorting (e.g. peanuts (Torres et al., 2014)) and, in case of cereals, after a certain degree of debranning, i.e. (partly) removing the outer layer of the kernel which is often more contaminated with mycotoxins. Common processing, such as fermentation, heating and household preparation may have a further effect. In their exposure assessment on deoxynivalenol (DON), Boon et al. (2009) applied a processing factor to occurrence data in raw wheat and rice samples to estimate the levels in prepared pasta and rice.

\section{Total diet study}

In total diet studies, food samples representing the whole diet and prepared as consumed are analysed for food chemicals. Samples are collected, prepared and pooled into composite samples per food category (EFSA/FAO/ WHO, 2011b; Marin et al., 2013). Due to the pooling of samples, results are not appropriate for compliance or law enforcement, although they may trigger follow-up studies. Samples collected within a total diet study should cover at least one of the two main aspects of representativeness: seasonality and geographical variation (EFSA/FAO/WHO, 2011a). The geographical coverage is important because of potential regional differences in dietary patterns and in the levels of mycotoxins in foods. Even if no differentiation is made in the exposure assessment between regions, the sampling has to cover potential geographical differences. For small countries like the Netherlands, this might be less relevant (Sprong et al., 2016b), but for larger countries with different climate zones of very different ethnic groups or large variations in consumption patterns, this must be carefully addressed (Australia New Zealand Food Standards, 2011). For locally produced foods, it is important to ensure that discount shops, supermarkets and farmers' markets are sampled proportionally to their market share. Seasonality should be addressed for foods in which the mycotoxin levels may vary due to climatic conditions (i.e. cereals) or seasonal supply variations.

The preparation of the food list is of paramount importance in total diet studies. The food list should cover around 90\% of the food intake, should be as close as possible to the actual whole diet and should include beverages and drinking water (EFSA/FAO/WHO, 2011b). Representative food items should be identified and selected from food consumption data. Food processing and at-home food preparation, like addition of water, salt and use of cooking utensils, should 
be selected for each type of food and should be as close as possible to the habits of the population. Finally, the degree of pooling into the composite samples should be determined according to the total diet study purposes. Pooling of the processed samples is an essential step and consists of creating a unique food sample (composite sample) for analysis. This is achieved by combining various individually prepared food items either of the same type (individual food approach; e.g. one apple sample made of different varieties of apples) or by mixing several different foods from the same food group (mixed food approach; e.g. one fruit sample made of different types of fruits like apple, pear or banana).

In a total diet-like study, the food items for which contamination levels of the relevant (group of) substances are expected are sampled separately (EFSA/FAO/WHO, 2011a). So in case of patulin, apple products, being the main source of this mycotoxin (Beretta et al., 2000), should be sampled and combined into a separate composite sample, not containing other fruit products (Sprong et al., 2016b). The same applies to aflatoxin $M_{1}$ which is only present in dairy products, while e.g. the occurrence of fumonisin $\mathrm{B}_{1}$ seems more limited to maize derived products and potentially wheat (Cendoya et al., 2014).

Total diet-like studies specifically designed for mycotoxin exposure were carried out in France in 2013 (Sirot et al., 2013) and in the Netherlands in 2014 (Lopez et al., 2016a; Sprong et al., 2016a,b). In the French study, 1,319 food items were collected, prepared and pooled, resulting in 577 composite samples for mycotoxin analysis. In the study in the Netherlands, 1,617 food items were pooled into 213 sub-samples, which were further pooled proportionally according to the consumption habits of the population in the Netherlands, eventually resulting in 88 composite samples for analysis (Sprong et al., 2016b).

Foods contributing most to the exposure can be identified by combining the analysed levels in the total diet(-like) study with food consumption data (Cano-Sancho et al., 2011; Leblanc et al., 2005; Sirot et al., 2013; Sprong et al., 2016a; Vin et al., 2014). EFSA distinguishes two types of total diet study approaches: a total diet study for screening, consisting of a limited number of composite food samples representative for common food categories, and a total diet study for refined exposure assessment containing a large number of samples representative for smaller, more refined, food categories (EFSA/FAO/WHO, 2011a). If the screening indicates high exposures, further evaluation should be performed to identify its source.

Storage of the samples before and after preparation of composites must be assessed, but for mycotoxins this seems less of an issue since they are stable in samples stored in the freezer and during freeze drying. Due to pooling of samples, and thus dilution of the compounds of interest, a low LOQ must be aimed at. Although multi-methods can be applied to the more generic samples, the specific composite samples need dedicated analysis with lower LOQs. For instance, Lopez et al. (2016a) applied a dedicated method for trichothecenes using GC-MS/MS after derivatisation, which resulted in ten times lower LOQs than those obtained with multi-methods (Lopez et al., 2016a; Sirot et al., 2013).

No volunteers are involved in total diet(-like) studies and hence no ethical issues are involved in the collection of the samples. However, approval from the competent ethics commission might be required when the study aims at diets of specific ethical groups in the population.

Costs lay in the design of the study, the preparation of the samples and the analysis of the samples. Costs of analysis can be high due to the low LOQs required and can be $50 \%$ of the total costs of the study (EFSA/FAO/WHO, 2011b). However, once the total diet study is performed, the design can be used to repeat the study for follow-up studies, thereby reducing their costs considerably. Samples stored may allow trend analysis of known and emerging mycotoxins for risk management information. However, samples collected specifically for certain mycotoxins, may be less useful for other classes of mycotoxins or other contaminants, since these may occur in other specific products that were not sampled separately and/or may be volatile and therefore lost during freeze-drying.

\section{Duplicate diet study}

In duplicate diet studies, an exact copy of all the foods and drinks as consumed by one person in a certain time period, e.g. 24 hours, is collected by volunteers with an accompanying food diary. In duplicate diet studies carried out in the Netherland this typically yields about $300 \mathrm{~g}$ of freeze dried samples per person. These studies are used to measure the actual exposure of consumers to compounds of interest, including the effects of food processing and preparation (Jekel and Van Egmond, 2014; Vaessen and Schothorst, 1999). Since samples are a mix of the foods as consumed, the contamination can seldom be traced to a specific food ingredient or food which makes the method unsuitable for enforcement of legislation, but allows comparing exposure data with health-based guidance values.

Several alternatives of the duplicate diet study are known, such as the cyclic sub-portion duplicate diet, the subpopulation duplicate diet, targeted food duplicate diet and the total population diet (Tomerlin et al., 2002). In these alternatives only a portion of the diet is collected or foods are collected based on standardised or average diets. Duplicate diets collected per eating event (Melnyk et al., 2012) allows the identification of a specific group of foods 
responsible for the main exposure. For example aflatoxin $M_{1}$ and fumonisins may occur more frequent in breakfast samples since dairy products (milk, yoghurt) and maize based cornflakes are, in many regions of the EU, consumed at breakfast. DON can be present in all meals containing cereal products, which are often consumed during the whole day in European type diets.

The study design is quite straightforward, but the sample collection and preparation are complex. As a result, duplicate diet studies are always limited in the number of participants and sampling days, which hampers the extrapolation of the results to larger populations.

The analysis of duplicate diets requires low LOQs due to dilution of the compounds in the sample (Jekel and Van Egmond, 2014). After homogenisation of the entire sample, it is typically freeze dried and stored in the freezer. Mycotoxins are stable under these conditions, which allows the collection of historic information (trends) when analysing emerging mycotoxins in the future. An example is the exposure assessment for T-2/HT-2 toxins in a study in the Netherlands (Jekel and Van Egmond, 2014).

The involvement of volunteers means that ethical issues must be addressed before the start of the collection. The actual collection of the duplicate diet typically involves the use of a cooled container which can be a burden to volunteers and may influence the diet consumed in the period of collection. Costs for collection of duplicate diets are high and only a limited number of samples can be collected. The latter reduces the costs for analysis but these can still be high due to the very low LOQs required.

\section{Exposure assessment strategies}

Exposure assessment is defined by the Food and Agriculture Organisation of the United Nations (FAO) and World Health Organisation (WHO) as the qualitative and/or quantitative evaluation of the likely intake of chemical agents via food as well as exposure from other sources if relevant (FAO/ WHO, 2008). For estimating the dietary exposure of humans to mycotoxins, information on prevalence and levels in foods are combined with consumption information (Brera et al., 2014; Cressey and Reeve, 2013; EFSA, 2014b). A schematic overview of the well-established exposure assessment strategies for among others mycotoxins is given in Figure 1 and the characteristics are described in Table 2. Each strategy yield unique information and has its own merits and challenges. Depending on the approach, these strategies may be used to estimate either acute exposure, i.e. during a period of 24 hours or less, or chronic exposure, i.e. over a longer period of time: from a couple of months up to several years.

\section{Point estimate}

In point estimate assessments, a single mycotoxin concentration value is combined with a single input parameter for consumption. The result is one single exposure estimate with a high degree of uncertainty. The input for concentration often comes from a food monitoring study or a total diet study. A mean or high consumption level from a food consumption survey is often used for input for consumption. If exposure via more than one food product is expected, the calculated exposures per food item can be combined to assess the total exposure.

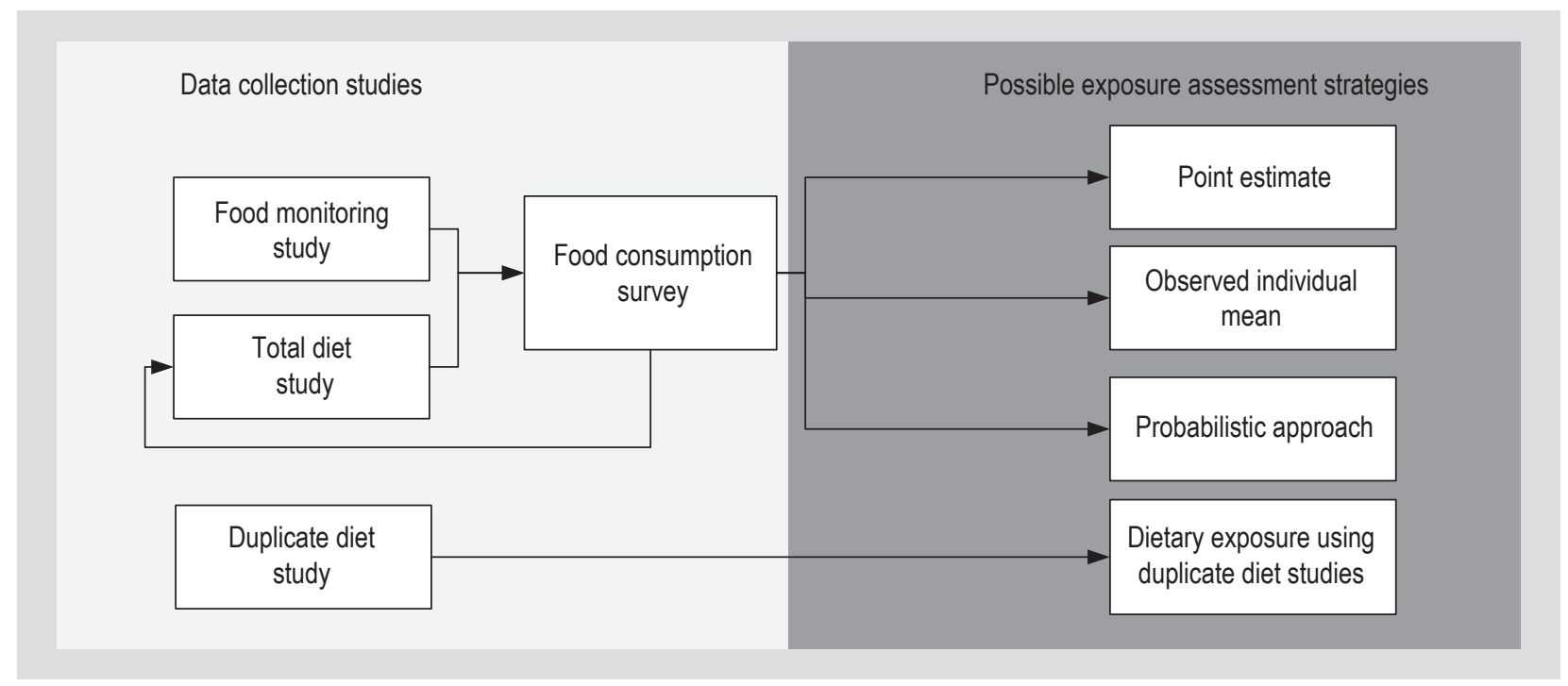

Figure 1. Overview of the methods for data collection and how these serve as input for different strategies to assess the dietary exposure. For total diet studies, the food list included should cover about $90 \%$ of the food intake and therefore knowledge of food consumption is a prerequisite for these studies (feed-back loop). 
Table 2. Main characteristics of four well-established exposure assessments methodologies.

\begin{tabular}{|c|c|c|c|c|c|c|c|c|}
\hline 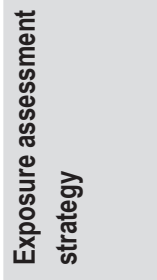 & 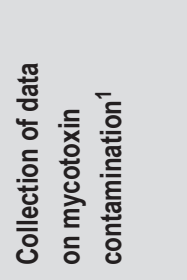 & 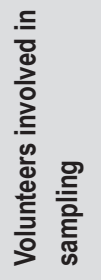 & 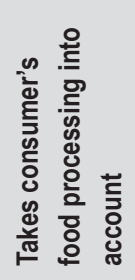 & 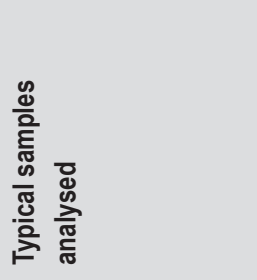 & 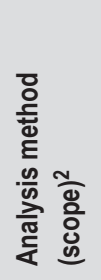 & 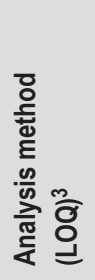 & 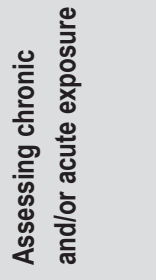 & 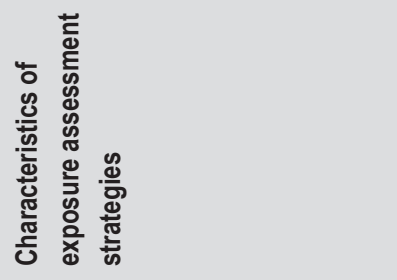 \\
\hline \multirow[t]{2}{*}{$\begin{array}{l}\text { Point } \\
\text { estimate }\end{array}$} & FMS & No & $\mathrm{No}^{4}$ & $\begin{array}{l}\text { Individual food } \\
\text { ingredients/products }\end{array}$ & + & $-1++$ & \multirow[t]{2}{*}{$\begin{array}{l}\text { Chronic and } \\
\text { acute }\end{array}$} & $\begin{array}{l}\text { Input: single concentration and } \\
\text { consumption level per food }\end{array}$ \\
\hline & $\begin{array}{l}\text { TDS (chronic) } \\
\text { combined } \\
\text { with data } \\
\text { from FCS }\end{array}$ & & Yes & $\begin{array}{l}\text { Composite processed } \\
\text { food products }\end{array}$ & $-1+$ & $++/-$ & & $\begin{array}{l}\text { Output: one chronic or acute intake } \\
\text { level (mean, high) } \\
\text { Easy to perform }\end{array}$ \\
\hline \multirow{2}{*}{$\begin{array}{l}\text { Observed } \\
\text { individual } \\
\text { mean }\end{array}$} & FMS & No & $\mathrm{No}^{4}$ & $\begin{array}{l}\text { Individual food } \\
\text { ingredients/products }\end{array}$ & + & $-1++$ & \multirow[t]{2}{*}{ Chronic } & $\begin{array}{l}\text { Input: distribution of consumption } \\
\text { and mean concentration levels }\end{array}$ \\
\hline & $\begin{array}{l}\text { TDS combined } \\
\text { with data } \\
\text { from FCS }\end{array}$ & & Yes & $\begin{array}{l}\text { Composite processed } \\
\text { food products }\end{array}$ & $-1+$ & $++/-$ & & $\begin{array}{l}\text { Output: distribution of mean intake } \\
\text { levels over all recording days per } \\
\text { individual } \\
\text { Computer modelling }\end{array}$ \\
\hline \multirow[t]{2}{*}{$\begin{array}{c}\text { Probabilistic } \\
\text { approach }\end{array}$} & FMS & No & $\mathrm{No}^{4}$ & $\begin{array}{l}\text { Individual food } \\
\text { ingredients/products }\end{array}$ & + & $-1++$ & \multirow[t]{2}{*}{$\begin{array}{l}\text { Chronic and } \\
\text { acute }\end{array}$} & $\begin{array}{l}\text { Input: as Observed Individual Mean } \\
\text { Output: distribution of chronic }\end{array}$ \\
\hline & $\begin{array}{l}\text { TDS (chronic) } \\
\text { combined } \\
\text { with data } \\
\text { from FCS }\end{array}$ & & Yes & $\begin{array}{l}\text { Composite processed } \\
\text { food products }\end{array}$ & $-1+$ & $++/-$ & & $\begin{array}{l}\text { intake, adjusted for within- } \\
\text { individual variation; distribution of } \\
\text { acute intake per person } \\
\text { Computer modelling }\end{array}$ \\
\hline $\begin{array}{l}\text { Duplicate } \\
\text { diet }\end{array}$ & DDS & Yes & Yes & $\begin{array}{l}\text { Composite processed } \\
\text { food products }\end{array}$ & - & ++ & $\begin{array}{l}\text { Chronic and } \\
\text { acute }\end{array}$ & $\begin{array}{l}\text { Input: duplicate portions of food } \\
\text { Output: measured intake per } \\
\text { duplicate portion (individual } \\
\text { exposure assessment) }\end{array}$ \\
\hline
\end{tabular}

The Joint FAO/WHO Expert Committee on Food Additives (JECFA, 2011) used this approach to calculate for example the chronic and acute exposure to DON and its acetylated derivatives. For chronic exposure, the average consumption data from 10 different regions (GEMS/food consumption cluster diets) were coupled with the weighted mean levels of DON and its acetyl metabolites in different food categories, and summed to obtain an overall estimate of the chronic exposure to DON and its acetyl metabolites per geographic region. In this example, for acute exposure, a wheat intake of $9 \mathrm{~g} / \mathrm{kg}$ bw (around the P97.5) was coupled to a high mycotoxin level of $10 \mathrm{mg} / \mathrm{kg}$ wheat.

In point estimates, often conservative estimates of the input variables are applied, resulting in high-end estimation of the exposure. The main advantage of this approach is that it is easy to perform and often used for a first conservative risk identification.

\section{Observed individual mean}

With the observed individual mean approach, a mean mycotoxin concentration per food product is combined with the food consumption per day per consumer, averaged over the days available in the survey and, in most cases, divided by the individual's body weight (Boon and Van der Voet, 2015). This results in the average exposure per $\mathrm{kg}$ bw per person per day. Results from a food monitoring study or a total diet study are used as input for mycotoxin concentration. 
For chronic exposure, the mean concentration of mycotoxins is used, because in the long run it is unlikely that a consumer will always consume products with only high or only low levels. Typically, information on food consumption is available over a period of 2 to 7 days per individual. With this approach, a distribution of daily average exposures for different individuals within a population is generated. From this distribution the mean and median chronic exposure and upper percentiles (e.g. $95^{\text {th }}$ or $97.5^{\text {th }}$ ) for that population can be estimated. This method is currently used by EFSA to assess the chronic exposure to food contaminants and additives (EFSA, 2012a,c; 2015c). In the case of chronic exposure to mycotoxins it was applied for ochratoxin A (EFSA, 2006), aflatoxins (EFSA, 2007), Alternaria toxins (EFSA, 2011a), T2/HT-2 toxins (EFSA, 2011b), zearalenone (EFSA, 2011c), ergot alkaloids (EFSA, 2012d), nivalenol (EFSA, 2013a), sterigmatocystin (EFSA, 2013b), DON (EFSA, 2013d), fumonisins (EFSA, 2014a) and beauvericin/ enniatins (EFSA, 2014b). Occurrence data from a total diet study were recently used by to assess the exposure of consumers in the Netherlands to 48 mycotoxins, including patulin, aflatoxins, ochratoxin A, fumonisins, zearalenone, trichothecenes, ergot alkaloids, Alternaria toxins, beauvericin and enniatins (Lopez et al., 2016a; Sprong et al., 2016a,b).

\section{Probabilistic approach}

With the use of the probabilistic approach, both the acute and chronic exposure via food can be estimated. The acute exposure is estimated by combining daily individual consumption patterns from a food consumption survey with randomly selected levels per food product from a databank with mycotoxin levels in individual samples. The resulting individual exposures per food product are summed to obtain the exposure per day and subsequently divided by the individual's body weight. This procedure is repeated multiple times (Monte Carlo simulation) resulting in individual daily exposure estimates that reflect all plausible combinations of daily consumptions and concentrations in a population. The upper part of the distribution represents consumers with a high intake of the compound (high consumers), which is important for assessing the acute risk.

In the case of ergot alkaloids, which may exert acute toxic effects, EFSA combined individual consumption data with a high mycotoxin level (P95 or the mean of the upper quartile) for the four main contributing food groups and an average level for the remainder (EFSA, 2012d). By summing up the exposure per individual per day, a distribution of so-called 'acute' exposure estimates was generated. Both the mean and P95 values for these distributions in the various food consumption surveys were calculated and used to assess acute risks. Since no individual mycotoxin levels in food are used in this study, this approach is merely a combination of the point estimate (fixed levels) and a probabilistic approach (distribution of consumption).

With the use of the probabilistic approach, also the chronic exposure can be estimated using statistical models which use the same input as the observed individual mean approach (see previous section). The largest uncertainty of the use of the observed individual mean approach to assess the long-term exposure is equalling the distribution of mean exposures over the person-days per person, typically two days, to the 'true' long-term exposure distribution of a given population. Given the limited number of persondays present in a food consumption database per person and the variation in daily food consumption patterns within an individual, the distribution of mean exposures over individuals obtained with the observed individual mean approach will often be too wide in comparison to distributions of 'true' long term exposures (Goedhart et $a l ., 2012)$. These distributions contain both the variation in exposure between individuals and between days for the same individual (the within individual variation), whereas for the long-run the variation within individuals is not relevant by definition (the long-term exposure distribution is the variation between individuals, not within individuals) (Boon and Van der Voet, 2015). Statistical models that separate these two sources of variation, and subsequently remove the within individual variation from the longterm exposure distribution, have proven to be useful for the estimation of long-term exposure (Dodd et al., 2006; Hoffmann et al., 2002; Slob, 1993).

The resulting long-term exposure distribution will be less broad than the distribution obtained with the observed individual mean approach, resulting especially in lower estimates of the higher percentiles of exposure and higher estimates of the lower percentiles. For example, the mean exposure assessed over just two days is more variable than the mean exposure assessed over more (up to hundreds) days that constitute a longer period of time.

\section{Dietary exposure using duplicate diet studies}

In duplicate diet studies (described in detail in Section 2) daily portions are analysed for the compound(s) in question, resulting in an actual exposure level per day for that individual. The food consumption data collected in accompanying food diaries can be used to evaluate the possible sources of exposure. When duplicate portions are collected on only one day per individual, these data can only be used to assess acute exposure. If duplicate portions are collected on multiple days, chronic exposure can be estimated by either averaging the exposure over the collection days per individual (see observed individual mean) or by removing the within individual variation using statistical models (see probability approach). Duplicate diets have been collected and analysed for estimating the 
exposure to various mycotoxins, like aflatoxin $\mathrm{B}_{1}$, aflatoxin $\mathrm{M}_{1}$, ochratoxin $\mathrm{A}$, trichothecenes, fumonisins and $\mathrm{T}-2 /$ HT-2 toxins (Bakker et al., 2009; Gilbert et al., 2001; Jekel and Van Egmond, 2014; Sizoo and Van Egmond, 2005).

\section{Human biomonitoring studies}

An alternative way to assess exposure to a compound is the use of biomarkers to quantify the internal dose. Characteristics of the data collection studies and subsequent exposure assessment strategy are mentioned in Table 3 and 4.

Exposure can be assessed by biomarkers of exposure. This includes the detection of the parent compound (mycotoxins) and/or its main phase I and phase II metabolites (e.g. glucuronide conjugates), measured in accessible body fluids (blood, urine) or body specimen (like hair). In contrast, biomarkers of effect can be used to assess the outcome after exposure to mycotoxins and include, e.g. changes in level of specific proteins (including diagnostic enzymes), cellular metabolites (metabolomics), or gene expression profiles (toxicogenomics) resulting from the specific alteration in metabolic or signalling pathways (Valencia-Quintana et al., 2014). Since these changes can also results from inter-individual differences, effects from other compounds in food or underlying diseases, it is difficult to link them exclusively to the intake of mycotoxins. Human biomarker research related to mycotoxins started in the early 1990's to gain insight into the mechanisms of action of aflatoxin $\mathrm{B}_{1}$ and to control the outcome of intervention strategies. Aflatoxin $\mathrm{M}_{1}$ was the predominant biomarker of exposure, detected in milk and urine during exposure assessments (Gambacorta et al., 2013; Routledge et al., 2014). Changes in the sphingosine/sphinganine ratio were used as biomarker of exposure and effect for fumonisins (Eaton and Gallagher, 1994; Gilbert et al., 2001; Guengerich et al., 1998; Mclean and Dutton, 1995; Shephard et al., 2007).

The measurement of biomarkers of exposure is the only available strategy that integrates exposure from all sources, (e.g. food, inhalation, skin contact) and reflects the internal biological active fraction (Choi et al., 2015). The exposure can be assessed by converting urinary mycotoxin concentrations to intake levels, taking into account their kinetics, such as rate of excretion (i.e. \% of the ingested mycotoxins excreted as the parent compound or metabolites in urine), daily urine production (defined by e.g. creatinine)

Table 3. Characteristics of: (1) study design; (2) sample number and analysis; (3) ethical issues; (4) costs; and (5) ability to use provided samples for other contaminants.

$\begin{aligned} & \text { Data collection } \\ & \text { study }\end{aligned}$
$\begin{array}{ll}\text { Human biomonitoring } & \text { 1. Study design is complex; knowledge on relevant biomarkers and toxicokinetics is required. } \\ \text { studies } & \text { 2. Collection and analysis involves handling of body fluids; high costs for sample preparation; standardised analytical methods not } \\ & \text { readily available; dedicated highly sensitive analysis required to achieve quantifiable results due to low concentrations in urine. } \\ & \text { 3. A representative group of reliable volunteers must be identified; ethical approval is required and privacy must be managed. } \\ & \text { 4. Relatively high analysis costs per sample/mycotoxin. } \\ & \text { 5. Samples can be used for analysis of multiple contaminants; allows estimation of chronic and acute exposure to } \\ & \text { contaminants. }\end{array}$

Table 4. Main characteristics of exposure assessments via biomarkers.

\begin{tabular}{|c|c|c|c|c|c|c|c|c|}
\hline $\begin{array}{l}\text { Exposure } \\
\text { assessment } \\
\text { strategy }\end{array}$ & $\begin{array}{l}\text { Collection } \\
\text { of data on } \\
\text { mycotoxin } \\
\text { contamination }{ }^{1}\end{array}$ & $\begin{array}{l}\text { Volunteers } \\
\text { involved in } \\
\text { sampling }\end{array}$ & $\begin{array}{l}\text { Takes consumer's } \\
\text { food processing } \\
\text { into account }\end{array}$ & $\begin{array}{l}\text { Typical } \\
\text { samples } \\
\text { analysed }\end{array}$ & $\begin{array}{l}\text { Analysis } \\
\text { method } \\
\text { (scope) }{ }^{2}\end{array}$ & $\begin{array}{l}\text { Analysis } \\
\text { method } \\
(\mathrm{LOQ})^{3}\end{array}$ & $\begin{array}{l}\text { Assessing } \\
\text { chronic and/ } \\
\text { or acute } \\
\text { exposure }\end{array}$ & $\begin{array}{l}\text { Characteristics } \\
\text { of exposure } \\
\text { assessment } \\
\text { strategies }\end{array}$ \\
\hline $\begin{array}{l}\text { Human } \\
\text { biomonitoring }\end{array}$ & HBS & Yes & Yes & Urine, blood & $-1+$ & ++ & $\begin{array}{l}\text { Chronic and } \\
\text { acute }\end{array}$ & $\begin{array}{l}\text { Input: concentrations } \\
\text { in biological fluids } \\
\text { Output: estimation of } \\
\text { uptake or intake }\end{array}$ \\
\hline
\end{tabular}


and body weight (Gratz et al., 2014; Rodriguez-Carrasco et al., 2014; Sarkanj et al., 2013; Solfrizzo et al., 2014; Turner et al., 2008; Wallin et al., 2013; Warth et al., 2012). This re-calculation is only possible for toxins showing a rapid elimination and short mean residence time (like for example DON). Since most of the toxicokinetic studies for mycotoxins have been performed in animals and specific data for humans are often lacking, exposure assessment using biomarkers of exposure is up to now a quantitative approach with high uncertainties.

Nevertheless, human biomonitoring was recently used to assess exposure of the Belgian population to the mycotoxins aflatoxins, citrinin, fumonisins, trichothecenes, ochratoxin A, zearalenone and their metabolites or modified forms (Heyndrickx et al., 2015). This study showed the very high prevalence of exposure to toxins such as DON. Moreover, citrinin was found in $50 \%$ of the urine samples while this mycotoxin was detected at only low levels and incidence in monitored food samples. This apparent discrepancy may result from variations in kinetic factors influencing the excretion of citrinin in humans, but may also indicate the need to identify as yet neglected sources of human citrinin exposure (Heyndrickx et al., 2015).

A human biomonitoring study may also give insight in the bioavailability of mycotoxins from unexpected or unknown sources and the presence of modified forms in food (e.g. glucosides, sulphates), which may become available for absorption after conversion to the parent compound in the gastrointestinal tract (EFSA, 2014a; Solfrizzo et al., 2014). These modified mycotoxins are those forms that may not be included in the currently applied analytical methods, partly since analytical standards and reference materials are not yet available or because the (modified) mycotoxins are not extracted with the applied methods.

In summary, although biomarkers for mycotoxin exposure can be measured in various biological fluids and tissues, such as (breast)milk, plasma, saliva, faeces, hair, nails, liver, kidneys or lungs, urine has become the preferred matrix (Sewram et al., 2001; Shephard et al., 2007). Human biomonitoring can identify vulnerable groups and can reduce the assumptions regarding consumption rates (Choi et al., 2015). Results of epidemiological studies on mycotoxins using biomonitoring have been reported in the past two decades (Duarte et al., 2012; Ediage et al., 2013; Gilbert et al., 2001; Pena et al., 2006; Rubert et al., 2011; Turner et al., 2010; Van der Westhuizen et al., 2011). These studies were based on only one urine sample per person, and thus the collection can be fairly easy (Heyndrickx et al., 2014; Solfrizzo et al., 2014). However, authors of a recent study, whereby the exposure to DON in three EU countries using these urinary biomarkers was assessed, strongly advice to collect 24-hours urine (Brera et al., 2015).
In order to compare the urinary concentrations within individuals, inter-individual variability in urine production needs to be circumvented. For this reason, urinary creatinine concentrations are widely used to adjust urinary concentrations of chemicals or their metabolites (Barr et al., 2005; Turner et al., 2010). Furthermore, the analysis of urine samples requires low LOQs, which can be reached by applying targeted analysis. Moreover, urine samples are usually treated with enzymes like $\beta$-glucuronidase/ sulfatase to convert conjugates into the parent compound (Solfrizzo et al., 2014). Costs for human biomonitoring studies are high due to the involvement of large numbers of volunteers, a complicated study design and sample collection. Analysis of the samples is costly. Finally, human biomonitoring involve volunteers and, therefore, ethical issues must always be addressed and a consensus of the volunteers is needed.

\section{Comparison of the different approaches}

\section{Selection of the most suitable approach}

In practice, a tiered approach can be used to estimate the exposure to mycotoxins within a population. In lower tiers, exposure estimates are based on limited data using a point estimate approach. When exposure seems to be significant, these estimates can be refined, in the case of chronic exposure using the observed individual mean and/or statistical methods for extrapolation to long-term exposure, and for acute exposure the probabilistic approach, resulting in the most accurate estimation of both types of intake (Pastoor et al., 2014).

Advantage of observed individual mean and probabilistic approaches is that the entire food consumption database, and food concentration data, is considered which results in more realistic exposure estimates of a given population (in a country of geographic region). This supports the derivation of a health-based guidance value and identifies segments of a population (such as children) with a higher risk. Another advantage is that also the uncertainty in the exposure estimations, caused by a limited size of the database, can be quantified (EFSA, 2012b).

The effects of food processing and preparation are included in total diet studies and in duplicate diet studies. This is of particular importance for less stable compounds, as otherwise exposure could be largely overestimated. An obvious limitation is the limited number of samples and the high costs and subject burden in duplicate diets studies. To illustrate this, in a duplicate diet study in the Netherlands into the exposure to T-2/HT-2 toxins covering several years, about 125 samples were collected per year (Jekel and Van Egmond, 2014). Subsequently, the duration of the duplicate diet studies is often limited to one day enabling acute, and much less for chronic, exposure assessment (Solfrizzo et al., 
2014). Drawback of a total diet study is the required large amount of food items. To illustrate this, 1,617 single food items were included in a total of 88 composite samples in a total diet study on exposure to mycotoxins (Sprong et al., 2016a). When assessing the exposure to mycotoxins using food consumption and food mycotoxin level data, effect of processing can be included by using processing factors.

Human biomonitoring is the only strategy that includes exposure from sources other than food, although this may be less relevant in the case of mycotoxins. Human biomonitoring requires a detailed understanding of the toxicokinetics in the human body which is still poorly understood for many mycotoxins. A clear advantage of biomarker studies is the estimation of the internal dose, determining the biological effects, and the identification of biomarkers that combine effect and exposure monitoring, such as DNA adducts of aflatoxins. Moreover, measuring the actual concentration in body fluids addresses the uncertainty of the existence of modified forms of mycotoxins that remain undetected by conventional analytical methods. Recently, EFSA concluded that glucosides of DON, nivalenol and zearalenone, and the hidden forms of fumonisins (EFSA, 2014a) may considerably contribute to the overall human exposure. In addition, EFSA proposed to apply relative potency factors for all known biologically active metabolites. This applies for example to zearalenone, as an equivalence factor of 60 needs to be considered for its metabolite $\alpha$-zearalenol, in the derivation of health-based guidance levels (EFSA, 2016).

\section{Emerging mycotoxins}

Exposure assessments of mycotoxins that have no legal limit face some additional challenges. At the moment of data collection, it may not be clear which foods contribute most to the exposure, samples may not be well characterised, analytical reference standards or reference materials are lacking, method of analysis may not be harmonised or validated, LOQs required to exclude potential health risks are unknown (Mol et al., 2015), and the relation between intake and excreted metabolites is unknown. Analysis of long-time stored duplicate or total diets may overcome these problems to some extent, assuming all relevant consumed foods are collected. When new mycotoxins are discovered, or new analytical methods with lower LOQs become available, or when applying trend analyses, these stored samples can provide valuable information (Jekel and Van Egmond, 2014). Human biomonitoring studies may have little relevance in this situation if relevant biomarkers of emerging mycotoxins are not yet defined.

\section{Trends in levels and exposure}

Ideally, food consumption surveys are regularly updated, to assess changes in mycotoxin exposure in time. Trends like an increased consumption of breakfast cereals containing nuts may increase the exposure to mycotoxins present in nuts, such as aflatoxins and ochratoxin A. Results from monitoring studies can be used for analysing trends in mycotoxin occurrence in (raw) food materials and may lead to an updated exposure assessment. Significant increases in mycotoxin exposure, even if below the healthbased guidance value, can be detected, with subsequent identification of the source of the increased exposure and evaluation of measures for reduction.

\section{Conclusions}

Exposure assessments of mycotoxins are complicated since their concentrations in food: (1) are often heterogeneously distributed over the samples; (2) differ between geographic regions and production years; (3) can be affected by (food) processing and preparation; and (4) mycotoxins can be present in modified forms. Each of the five strategies for estimating the dietary exposure of a population to mycotoxins discussed in this paper has its merits and restrictions. The same applies to the studies collecting the data needed to perform these assessments. A combination of the different strategies has an added value for the risk assessment of mycotoxins, including the identification of unknown sources or modified forms.

\section{Acknowledgements}

The authors would like to thank Prof. Em. Dr. J. FinkGremmels of the Utrecht University, Faculty of Veterinary Medicine, Institute for Risk Assessment Sciences, Division of Veterinary Pharmacology, Pharmacotherapy and Toxicology, for critically reviewing this paper. This study was partly carried out in WOT project-02-001-061 'Method development and surveys on mycotoxins in food' at RIKILT Wageningen UR, on behalf of and funded by the Ministry of Economic Affairs, the Netherlands. EH is financially supported by the Research Foundation Flanders (grant number G.0D4615.N) as part of 'The Food Biomarkers Alliance project (FOODBALL)' within the 'EU Joint Programming Initiative: A Healthy Diet for a Healthy Life: Biomarkers for Nutrition and Health' 


\section{References}

Australia New Zealand Food Standards, 2011. The $23^{\text {rd }}$ Australian total diet study. Food Standards Australia, pp. 127.

Bakker, G.J.I., Sizoo, E.A., Jekel, A.A., Pereboom-de Fauw, D.P.K.H., Schothorst, R.C. and Van Egmond, H.P., 2009. Determination of mean daily intakes of aflatoxin $B_{1}$, aflatoxin $M_{1}$, ochratoxin $A$, trichothecenes and fumonisins in 24-hour diets of children in the Netherlands. World Mycotoxin Journal 2: 451-459.

Barr, D.B., Wilder, L.C., Caudill, S.P., Gonzalez, A.J., Needham, L.L. and Pirkle, J.L., 2005. Urinary creatinine concentrations in the US population: implications for urinary biologic monitoring measurements. Environmental Health Perspectives 113: 192-200.

Battilani, P., Rossi, V., Giorni, P., Pietri A, Gualla, A., Van der Fels-Klerx, H.J., Booij, C.J.H., Moretti, A., Logrieco, A., Miglietta, F., Toscano, P., Miraglia, M., De Sanits, B. and Brera, C., 2012. Modelling, predicting and mapping the emergence of aflatoxins in cereals in the EU due to climate change MODMAP-AFLA. EFSA Supporting Publications 9: 1-172.

Beretta, B., Gaiaschi, A., Galli, C.L. and Restani, P., 2000. Patulin in apple-based foods: occurrence and safety evaluation. Food Additives and Contaminants 17: 399-406.

Boon, P.E., Bakker, M.I., Van Klaveren, J.D. and Van Rossum, C.T.M., 2009. Risk assessment of the dietary exposure to contaminants and pesticide residues in young children in the Netherlands. RIVM Report 350070002. National Institute for Public Health and the Environment (RIVM), Bilthoven, the Netherlands.

Boon, P.E. and Van der Voet, H., 2015. Probabilistic dietary exposure models. RIVM Letter report 2015-0085. National Institute for Public Health and the Environment (RIVM), Bilthoven, the Netherlands.

Brera, C., Debegnach, F., De Santis, B., Di Ianni, S., Gregori, E., Neuhold, S. and Valitutti, F., 2014. Exposure assessment to mycotoxins in gluten-free diet for celiac patients. Food and Chemical Toxicology 69: 13-17.

Brera, P., De Santis, B., Debegnach, F., Miano, B., Moretti, G., Lanzone, A., Del Sordo, G., Buonsenso, D., Chiaretti, A., Hardie, L., White, K., Brantseater, A.L., Knutsen, H., Eriksen, G.S., Sandvik, M., Wells, L., Allen, S. and Sathyapalan, T., 2015. Experimental study of deoxynivalenol biomarkers in urine GP/EFSA/CONTAM/2013/04. EFSA Supporting Publications EN-818: 136. EFSA, Parma, Italy.

Cano-Sancho, G., Gauchi, J.P., Sanchis, V., Marin, S. and Ramos, A.J., 2011. Quantitative dietary exposure assessment of the Catalonian population (Spain) to the mycotoxin deoxynivalenol. Food Additives and Contaminants Part A 28: 1098-1109.

Cano-Sancho, G., Sanchis, V., Ramos, A.J. and Marin, S., 2013. Effect of food processing on exposure assessment studies with mycotoxins. Food Additives and Contaminants Part A 30: 867-875.

Cendoya, E., Monge, M.P., Palacios, S.A., Chiacchiera, S.M., Torres, A.M., Farnochi, M.C. and Ramirez, M.L., 2014. Fumonisin occurrence in naturally contaminated wheat grain harvested in Argentina. Food Control 37: 56-61.

Choi, J., Morck, T.A., Polcher, A., Knudsen, L.E. and Joas, A., 2015. Review of the state of the art of human biomonitoring for chemical substances and its application to human exposure assessment for food safety. EFSA Supporting Publications EN-724: 321. EFSA, Parma, Italy.
Cressey, P.J., 2009. Mycotoxin risk management in New Zealand and Australian food. World Mycotoxin Journal 2: 113-118.

Cressey, P.J. and Reeve, J., 2013. Dietary exposure and risk assessment for aflatoxins in New Zealand. World Mycotoxin Journal 6: 427-437. De Nijs, M., Pereboom-de Fauw, D.P., Van Dam, R.C., De Rijk, T.C., Van Egmond, H.P. and Mol, H.J., 2013. Development and validation of an LC-MS/MS method for the detection of phomopsin A in lupin and lupin-containing retail food samples from the Netherlands. Food Additives and Contaminants Part A 30: 1819-1826.

De Rijk, T.C., Van Egmond, H.P., Van der Fels-Klerx, H.J., Herbes, R., De Nijs, M., Samson, R.A., Slate, A.B. and Van der Spiegel, M., 2015. A study of the 2013 western European issue of aflatoxin contamination of maize from the Balkan area. World Mycotoxin Journal 8: 641-651.

Dodd, K.W., Guenther, P.M., Freedman, L.S., Subar, A.F., Kipnis, V., Midthune, D., Tooze, J.A. and Krebs-Smith, S.M., 2006. Statistical methods for estimating usual intake of nutrients and foods: a review of the theory. Journal of the American Dietetic Association 106: 1640-1650.

Duarte, S.C., Alves, M.R., Pena, A. and Lino, C.M., 2012. Determinants of ochratoxin A exposure - a one year follow-up study of urine levels. International Journal of Hygiene and Environmental Health 215: 360-367.

Eaton, D.L. and Gallagher, E.P., 1994. Mechanism of aflatoxin carcinogenesis. Annual Review of Pharmacology and Toxicology 34: $135-172$

Ediage, E.N., Di Mavungu, J.D., Song, S.Q., Sioen, I. and De Saeger, S., 2013. Multimycotoxin analysis in urines to assess infant exposure: a case study in Cameroon. Environment International 57-58: 50-59.

European Commission (EC), 2004. Regulation (EC) No 882/2004 of the European Parliament and of the Council of 29 April 2004 on official controls performed to ensure the verification of compliance with feed and food law, animal health and animal welfare rules. Official Journal of the European Union L165: 1-141.

European Food Safety Authority (EFSA), 2006. Opinion of the scientific panel on contaminants in the food chain on a request from the commission related to ochratoxin A in food (Question $\mathrm{N}^{\circ}$ EFSA-Q-2005-154). EFSA Journal 365: 56.

European Food Safety Authority (EFSA), 2007. Opinion of the scientific panel on contaminants in the food chain on a request from the commission related to the potential increase of consumer health risk by a possible increase of the existing maximum levels for aflatoxins in almonds, hazelnuts and pistachios and derived products (Question N EFSA-Q-2006-174). EFSA Journal 446: 127.

European Food Safety Authority (EFSA), 2010. Management of left-censored data in dietary exposure assessment of chemical substances. EFSA Journal 8: 1557.

European Food Safety Authority (EFSA), 2011a. Scientific opinion on the risks for animal and public health related to the presence of Alternaria toxins in feed and food. EFSA Panel on Contaminants in the Food Chain. EFSA Journal 9: 2407.

European Food Safety Authority (EFSA), 2011b. Scientific opinion on the risks for animal and public health related to the presence of T-2 and HT-2 toxin in food and feed. EFSA Panel on Contaminants in the Food Chain. EFSA Journal 9: 2481. 
European Food Safety Authority (EFSA), 2011c. Scientific opinion on the risks for public health related to the presence of zearalenone in food. EFSA Panel on Contaminants in the Food Chain. EFSA Journal 9: 2197.

European Food Safety Authority (EFSA), 2011d. Use of the EFSA comprehensive European food consumption database in exposure assessment. EFSA Journal 9: 2097.

European Food Safety Authority (EFSA), 2012a. Cadmium dietary exposure in the European population. EFSA Journal 10: 2551.

European Food Safety Authority (EFSA), 2012b. Guidance on the use of probabilistic methodology for modelling dietary exposure to pesticide residues. EFSA Panel on Plant Protection Products and their Residues. EFSA Journal 10: 2839.

European Food Safety Authority (EFSA), 2012c. Lead dietary exposure in the European population. EFSA Journal 10: 2831.

European Food Safety Authority (EFSA), 2012d. Scientific opinion on ergot alkaloids in food and feed. EFSA Panel on Contaminants in the Food Chain. EFSA Journal 10: 2798.

European Food Safety Authority (EFSA), 2013a. Scientific opinion on risks for animal and public health related to the presence of nivalenol in food and feed. EFSA Panel on Contaminants in the Food Chain. EFSA Journal 11: 3262.

European Food Safety Authority (EFSA), 2013b. Scientific opinion on the risk for public and animal health related to the presence of sterigmatocystin in food and feed. EFSA Panel on Contaminants in the Food Chain. EFSA Journal 11: 3254.

European Food Safety Authority (EFSA), 2013c. Standard sample description ver. 2.0. EFSA Journal 11: 3424.

European Food Safety Authority (EFSA), 2013d. Statement on the risks for public health related to a possible increase of the maximum level of deoxynivalenol for certain semi-processed cereal products. EFSA Panel on Contaminants in the Food Chain. EFSA Journal 11: 3490.

European Food Safety Authority (EFSA), 2014a. Scientific opinion on the risks for human and animal health related to the presence of modified forms of certain mycotoxins in food and feed. EFSA Panel on Contaminants in the Food Chain. EFSA Journal 12: 3916.

European Food Safety Authority (EFSA), 2014b. Scientific opinion on the risks to human and animal health related to the presence of beauvericin and enniatins in food and feed. EFSA Panel on Contaminants in the Food Chain. EFSA Journal 12: 2902.

European Food Safety Authority (EFSA), 2015a. The EFSA comprehensive European food consumption database. Available at: http://www.efsa.europa.eu/en/food-consumption/comprehensivedatabase.

European Food Safety Authority (EFSA), 2015b. Major update boosts food consumption database. Available at: http://www.efsa.europa. $\mathrm{eu} / \mathrm{en} / \mathrm{press} /$ news/150428.

European Food Safety Authority (EFSA), 2015c. Statement of EFSA refined exposure assessment for quinoline yellow (E 104). EFSA Journal 13: 4070.

European Food Safety Authority (EFSA), 2016. Appropriateness to set a group health-based guidance value for zearalenone and its modified forms. EFSA Panel on Contaminants in the Food Chain. EFSA Journal 14: 4425.
European Food Safety Authority/Food and Agriculture Organization/ World Health Organization (EFSA/FAO/WHO), 2011a. State of the art on total diet studies based on the replies to the EFSA/FAO/WHO questionnaire on national total diet study approaches. Supporting Publications 206.

European Food Safety Authority/Food and Agriculture Organization/ World Health Organization (EFSA/FAO/WHO), 2011b. Towards a harmonised total diet study approach: a guidance document. EFSA Journal 9: 2450.

Food and Agriculture Organization/World Health Organization (FAO/ WHO), 2008. Codex Alimentarius commisssion procedural manual. Joint FAO/WHO Food Standards Programme. 186 pp.

Food and Agriculture Organization/World Health Organization (FAO/ WHO), 2009. Principles and methods for the risk assessment of chemicals in food. Environmental Health Criteria 240. Available at: http://www.who.int/foodsafety/publications/chemical-food/en/.

Food and Agriculture Organization/World Health Organization (FAO/WHO), 2012. Safety evaluations of certain food additives and contaminants. WHO Food Additives Series 65: 1-833.

Food and Agriculture Organization/World Health Organization (FAO/ WHO), 2014. Guidance document on international estimated short-term intake (IESTI). Available at: http://tinyurl.com/h3ttwne.

Food and Agriculture Organization/World Health Organization (FAO/ WHO), 2015. Chronic individual food consumption summary statistics. Available at: http://tinyurl.com/gm5cn5x.

Gambacorta, L., Solfrizzo, M., Visconti, A., Powers, S., Cossalter, A.M., Pinton, P. and Oswald, I.P., 2013. Validation study on urinary biomarkers of exposure for aflatoxin $\mathrm{B}_{1}$, ochratoxin $\mathrm{A}$, fumonisin $\mathrm{B}_{1}$, deoxynivalenol and zearalenone in piglets. World Mycotoxin Journal 6: 299-308.

Garcia-Cela, E., Ramos, A.J., Sanchis, V. and Marin, S., 2013. Risk management towards food safety objective achievement regarding to mycotoxins in pistachio: the sampling and measurement uncertainty issue. Food Control 31: 392-402.

Gilbert, J., Brereton, P. and MacDonald, S., 2001. Assessment of dietary exposure to ochratoxin A in the UK using a duplicate diet approach and analysis of urine and plasma samples. Food Additives and Contaminants 18: 1088-1093.

Goedhart, P.W., Van der Voet, H., Knuppel, S., Dekkers, A.L.M., Dodd, K.W., Boeing, H. and Van Klaveren, J., 2012. A comparison by simulation of different methods to estimate the usual intake distribution for episodically consumed foods. EFSA Supporting Publication EN-299: 1-65.

Gratz, S.W., Richardson, A.J., Duncan, G. and Holtrop, G., 2014. Annual variation of dietary deoxynivalenol exposure during years of different Fusarium prevalence: a pilot biomonitoring study. Food Additives and Contaminants Part A 31: 1579-1585.

Guengerich, F.P., Johnson, W.W., Shimada, T., Ueng, Y.F., Yamazaki, H. and Langouet, S., 1998. Activation and detoxication of aflatoxin $\mathrm{B}_{1}$. Mutation Research 402: 121-128.

Han, Z., Nie, D., Ediage, E.N., Yang, X., Wang, J., Chen, B., Li, S., On, S.L., De Saeger, S. and Wu, A., 2014. Cumulative health risk assessment of co-occurring mycotoxins of deoxynivalenol and its acetyl derivatives in wheat and maize: case study, Shanghai, China. Food and Chemical Toxicology 74: 334-342. 
Heyndrickx, E., Sioen, I., Bellemans, M., De Maeyer, M., Callebaut, A., De Henauw, S. and De Saeger, S., 2014. Assessment of mycotoxin exposure in the Belgian population using biomarkers: aim, design and methods of the BIOMYCO study. Food Additives and Contaminants Part A 31: 924-931.

Heyndrickx, E., Sioen, I., Huybrechts, B., Callebaut, A., De Henauw, S. and De Saeger, S., 2015. Human biomonitoring of multiple mycotoxins in the Belgian population: results of the BIOMYCO study. Environment International 84: 82-89.

Hoffmann, K., Boeing, H., Dufour, A., Volatier, J.L., Telman, J., Virtanen, M., Becker, W., De Henauw, S. and Efcosum Grp, 2002. Estimating the distribution of usual dietary intake by short-term measurements. European Journal of Clinical Nutrition 56: S53-S62.

Joint FAO/WHO Expert Committee on Food Additives (JECFA), 2011. Evaluation of certain contaminants in food - seventy-second report of the joint FAO/WHO expert committee on food additives. WHO Technical Report Series 959: 115.

Jekel, A.A. and Van Egmond, H.P., 2014. Determination of T-2/HT-2 toxins in duplicate diets in the Netherlands by GC-MS/MS: method development and estimation of human exposure. World Mycotoxin Journal 7: 267-276.

Kendra, D.F. and Dyer, R.B., 2007. Opportunities for biotechnology and policy regarding mycotoxin issues in international trade. International Journal of Food Microbiology 119: 147-151.

Leblanc, J.C., Tard, A., Volatier, J.L. and Verger, P., 2005. Estimated dietary exposure to principal food mycotoxins from The First French Total Diet Study. Food Additives and Contaminants 22: 652-672.

Lopez, P., De Rijk, T., Sprong, R.C., Mengelers, M.J.B., Castenmiller, J.J.M. and Alewijn, M., 2016a. A mycotoxin-dedicated total diet study in the Netherlands in 2013: part 2 - occurrence. World Mycotoxin Journal 9: 89-108.

Lopez, P., Venema, D., De Rijk, T., De Kok, A., Scholten, J.M. and De Nijs, M., 2016b. Occurrence of Alternaria toxins in food products in the Netherlands. Food Control 60: 196-204.

Marin, S., Ramos, A.J., Cano-Sancho, G. and Sanchis, V., 2013. Mycotoxins: occurrence, toxicology, and exposure assessment. Food and Chemical Toxicology 60: 218-237.

Mclean, M. and Dutton, M.F., 1995. Cellular interactions and metabolism of aflatoxin - an update. Pharmacology and Therapeutics 65: 163-192.

Meca, G., Zhou, T., Li, X.Z. and Manes, J., 2013. Beauvericin degradation during bread and beer making. Food Control 34: 8 .

Melnyk, L.J., McCombs, M., Brown, G.G., Raymer, J., Nishioka, M., Buehler, S., Freeman, N. and Michael, L.C., 2012. Community duplicate diet methodology: a new tool for estimating dietary exposures to pesticides. Journal of Environmental Monitoring 14: 85-93.

Mol, H.G.J., Pietri, A., MacDonald, S.J., Anagnostopoulos, C. and Spanjer, M., 2015. Survey on sterigmatocystin in food. EFSA Supporting Publication EN-774: 1-56.

Muller, M.E.H. and Korn, U., 2013. Alternaria mycotoxins in wheat - a 10 years survey in the Northeast of Germany. Food Control 34: 191-197.

O'Mahony, C. and Vilone, G., 2013. Compiled European food consumption database. Supporting Publications EN-415: 1-31.
Pastoor, T.P., Bachman, A.N., Bell, D.R., Cohen, S.M., Dellarco, M., Dewhurst, I.C., Doe, J.E., Doerrer, N.G., Embry, M.R., Hines, R.N., Moretto, A., Phillips, R.D., Rowlands, J.C., Tanir, J.Y., Wolf, D.C. and Boobis, A.R., 2014. A $21^{\text {st }}$ century roadmap for human health risk assessment. Critical Reviews in Toxicology 44: 1-5.

Pena, A., Seifrtova, M., Lino, C., Silveira, I. and Solich, P., 2006. Estimation of ochratoxin A in Portuguese population: new data on the occurrence in human urine by high performance liquid chromatography with fluorescence detection. Food and Chemical Toxicology 44: 1449-1454.

Pichler, E., 2013. Sampling for mycotoxins - do we care enough? Cereal Foods World 58: 117-118.

Rodriguez-Carrasco, Y., Molto, J.C., Manes, J. and Berrada, H., 2014. Exposure assessment approach through mycotoxin/creatinine ratio evaluation in urine by GC-MS/MS. Food and Chemical Toxicology 72: 69-75.

Routledge, M.N., Kimanya, M.E., Shirima, C.P., Wild, C.P. and Gong, Y.Y., 2014. Quantitative correlation of aflatoxin biomarker with dietary intake of aflatoxin in Tanzanian children. Biomarkers 19: 430-435.

Rubert, J., Soriano, J.M., Manes, J. and Soler, C., 2011. Rapid mycotoxin analysis in human urine: a pilot study. Food and Chemical Toxicology 49: 2299-2304.

Sanders, M., Landschoot, S., Audenaert, K., Haesaert, G., Eeckhout, M. and De Saeger, S., 2014. Deoxynivalenol content in wheat dust versus wheat grain: a comparative study. World Mycotoxin Journal 7: 285-290.

Sarkanj, B., Warth, B., Uhlig, S., Abia, W.A., Sulyok, M., Klapec, T., Krska, R. and Banjari, I., 2013. Urinary analysis reveals high deoxynivalenol exposure in pregnant women from Croatia. Food and Chemical Toxicology 62: 231-237.

Schwartzbord, J.R. and Brown, D.L., 2015. Aflatoxin contamination in Haitian peanut products and maize and the safety of oil processed from contaminated peanuts. Food Control 56: 114-118.

Sewram, V., Nair, J.J., Nieuwoudt, T.W., Gelderblom, W.C.A., Marasas, W.F.O. and Shephard, G.S., 2001. Assessing chronic exposure to fumonisin mycotoxins: The use of hair as a suitable noninvasive matrix. Journal of Analytical Toxicology 25: 450-455.

Shephard, G.S., Van der Westhuizen, L. and Sewram, V., 2007. Biomarkers of exposure to fumonisin mycotoxins: a review. Food Additives and Contaminants 24: 1196-1201.

Sirot, V., Fremy, J.M. and Leblanc, J.C., 2013. Dietary exposure to mycotoxins and health risk assessment in the second French total diet study. Food and Chemical Toxicology 52: 1-11.

Sizoo, E.A. and Van Egmond, H.P., 2005. Analysis of duplicate 24-hour diet samples for aflatoxin $B_{1}$, aflatoxin $M_{1}$ and ochratoxin $A$. Food Additives and Contaminants 22: 163-172.

Slob, W., 1993. Modeling long-term exposure of the whole population to chemicals in food. Risk Analysis 13: 525-530.

Solfrizzo, M., Gambacorta, L. and Visconti, A., 2014. Assessment of multi-mycotoxin exposure in southern Italy by urinary multibiomarker determination. Toxins 6: 523-538.

Spanjer, M.C., Scholten, J.M., Kastrup, S., Jorissen, U., Schatzki, T.F. and Toyofuku, N., 2006. Sample comminution for mycotoxin analysis: dry milling or slurry mixing? Food Additives and Contaminants 23: 73-83. 
Sprong, R.C., De Wit-Bos, L., Te Biesebeek, J.D., Alewijn, M., Lopez, P. and Mengelers, M.J.B., 2016a. A mycotoxin-dedicated total diet study in the Netherlands in 2013, part 3 - exposure and risk assessment. World Mycotoxin Journal 9: 109-127.

Sprong, R.C., De Wit-Bos, L., Zeilmaker, M.J., Alewijn, M., Castenmiller, J.J.M. and Mengelers, M.J.B., 2016b. A mycotoxindedicated total diet study in the Netherlands in 2013, part 1 - design. World Mycotoxin Journal 9: 73-87.

Straumfors, A., Uhlig, S., Eriksen, G.S., Heldal, K.K., Eduard, W., Krska, R. and Sulyok, M., 2015. Mycotoxins and other fungal metabolites in grain dust from Norwegian grain elevators and compound feed mills. World Mycotoxin Journal 8: 361-373.

Sulyok, M., Krska, R. and Schuhmacher, R., 2010. Application of an LCMS/MS based multi-mycotoxin method for the semi-quantitative determination of mycotoxins occurring in different types of food infected by moulds. Food Chemistry 119: 408-416.

Tittlemier, S.A., Roscoe, M., Trelka, R., Patrick, S.K., Bamforth, J.M., Grafenhan, T., Schlichting, L. and Fu, B.X., 2014. Fate of moniliformin during milling of Canadian Durum wheat, processing, and cooking of spaghetti. Canadian Journal of Plant Science 94: 555-563.

Tomerlin, J.R., Barraj, L.M., Melnyk, L., Berry, M.R. and Gordon, S.M., 2002. Alternatives to duplicate diet methodology. Epidemiology 13: S218-S219.

Torres, A.M., Barros, G.G., Palacios, S.A., Chulze, S.N. and Battilani, P., 2014. Review on pre- and post-harvest management of peanuts to minimize aflatoxin contamination. Food Research International 62: $11-19$

Turner, P.C., Hopton, R.P., Lecluse, Y., White, K.L., Fisher, J. and Lebailly, P., 2010. Determinants of urinary deoxynivalenol and de-epoxy deoxynivalenol in male farmers from Normandy, France. Journal of Agricultural and Food Chemistry 58: 5206-5212.

Turner, P.C., Rothwell, J.A., White, K.L.M., Gong, Y., Cade, J.E. and Wild, C.P., 2008. Urinary deoxynivalenol is correlated with cereal intake in individuals from the United Kingdom. Environmental Health Perspectives 116: 21-25.

Vaessen, H.A.M.G. and Schothorst, R.C., 1999. The oral nitrate and nitrite intake in the Netherlands: evaluation of the results obtained by HPIC analysis of duplicate 24-hour diet samples collected in 1994. Food Additives and Contaminants 16: 181-188.
Valencia-Quintana, R., Sanchez-Alarcon, J., Tenorio-Arvide, M.G., Deng, Y.J., Montiel-Gonzalez, J.M.R., Gomez-Arroyo, S., VillalobosPietrini, R., Cortes-Eslava, J., Flores-Marquez, A.R. and ArenasHuertero, F., 2014. The microRNAs as potential biomarkers for predicting the onset of aflatoxin exposure in human beings: a review. Frontiers in Microbiology 5: 1-14.

Van der Fels-Klerx, H.J., 2014. Evaluation of performance of predictive models for deoxynivalenol in wheat. Risk Analysis 34: 380-390.

Van der Westhuizen, L., Shephard, G.S., Burger, H.M., Rheeder, J.P., Gelderblom, W.C.A., Wild, C.P. and Gong, Y.Y., 2011. Fumonisin $\mathrm{B}_{1}$ as a urinary biomarker of exposure in a maize intervention study among South African subsistence farmers. Cancer Epidemiology, Biomarkers and Prevention 20: 483-489.

Van Egmond, H.P., Schothorst, R.C. and Jonker, M.A., 2007. Regulations relating to mycotoxins in food: perspectives in a global and European context. Analytical and Bioanalytical Chemistry 389: 147-157.

Vidal, A., Marin, S., Morales, H., Ramos, A.J. and Sanchis, V., 2014. The fate of deoxynivalenol and ochratoxin A during the breadmaking process, effects of sourdough use and bran content. Food and Chemical Toxicology 68: 53-60.

Vin, K., Papadopoulos, A., Cubadda, F., Aureli, F., Basegmez, H.I.O., D’Amato, M., De Coster, S., D'Evoli, L., Esteban, M.T.L., Jurkovic, M., Lucarini, M., Ozer, H., San Juan, P.M.F., Sioen, I., Sokolic, D., Turrini, A. and Sirot, V., 2014. TDS exposure project: relevance of the Total Diet Study approach for different groups of substances. Food and Chemical Toxicology 73: 21-34.

Wallin, S., Hardie, L.J., Kotova, N., Lemming, E.W., Nalsen, C., Ridefelt, P., Turner, P.C., White, K.L.M. and Olsen, M., 2013. Biomonitoring study of deoxynivalenol exposure and association with typical cereal consumption in Swedish adults. World Mycotoxin Journal 6: 439-448.

Warth, B., Sulyok, M., Fruhmann, P., Berthiller, F., Schuhmacher, R., Hametner, C., Adam, G., Frohlich, J. and Krska, R., 2012. Assessment of human deoxynivalenol exposure using an LC-MS/MS based biomarker method. Toxicology Letters 211: 85-90.

World Health Organization (WHO), 2015. GEMS/Food consumption database and the FAO/WHO chronic individual food consumption database - summary statistics (CIFOCOss). Available at: http:// www.who.int/foodsafety/databases/en/. 
